\title{
Semantic Maps Capturing Organization Knowledge in e-Learning
}

\author{
Androklis Mavridis ${ }^{1}$, Adamantios Koumpis ${ }^{1}$, and Stavros N. Demetriadis ${ }^{2}$ \\ ${ }^{1}$ Altec research programmes division, M. Kalou 6 str. \\ 54629 Thessaloniki, Greece \\ \{mavr, akoula $\}$ altec.gr \\ ${ }^{2}$ Informatics Department, Aristotle University of Thessaloniki, Greece \\ sdemetriacsd.auth.gr
}

\begin{abstract}
. e-learning, shows much promise in accessibility and opportunity to learn, due to its asynchronous nature and its ability to transmit knowledge fast and effectively. However without a universal standard for online learning and teaching, many systems are proclaimed as "e-learning-compliant", offering nothing more than automated services for delivering courses online, providing no additional enhancement to reusability and learner personalization. Hence, the focus is not on providing reusable and learner-centered content, but on developing the technology aspects of e-learning. This current trend has made it crucial to find a more refined definition of what constitutes knowledge in the e-learning context. We propose an e-learning system architecture that makes use of a knowledge model to facilitate continuous dialogue and inquiry-based knowledge learning, by exploiting the full benefits of the semantic web as a medium capable for supplying the web with formalized knowledge.
\end{abstract}

Keywords: e-Learning, Semantic Web, Knowledge maps.

\section{Introduction}

Due to its asynchronous nature, e-learning, shows much promise in accessibility and opportunity to learn. As e-learning can transmit knowledge fast and effectively, it is accepted by many as a means of upgrading themselves and keeping up with the rapid changes that define the Internet.

A literature review [1] unveiled major concerns about its effectiveness and appropriateness. Without a universal standard for online learning and teaching, many organizations proclaiming their systems as "e-learning-compliant", but actually these organizations are only automating their services and delivering their courses online. Except for the elimination of the time and space barrier, the online content provides no additional enhancement to the educational learning experience, and the reusability and learner personalization are not realized, as these organizations are developing elearning resources to suit their own contexts and using tools that hinder collaboration and reuse.

The focus is not on designing reusable and learner-centred content, as many developers are placing much emphasis on the technology aspects of e-learning [2]. This 
inevitably lead to an unfortunate situation where most content developers are concerned with showcasing their technology-enhanced products, showing little interest in enhancing the "knowledge aspect" of e-learning, which should be at the centre of it. This current trend coupled with the growth in access to ever increasing amounts of information, has made it crucial to find a more refined definition of what constitutes knowledge in the e-learning context. The emphasis should not be placed on the information on the web (as this does not constitute knowledge) but on managing information to transform it into knowledge.

In this paper we focus on these issues and we propose an e-learning system architecture that makes use of a knowledge model to facilitate continuous dialogue and inquiry-based knowledge learning in business environments. This architecture offers a knowledge environment that represents the semantic web version of e-learning, a view clearly reflected in the EU-NSF Strategic Workshop Report 2001, where it is observed that e-learning, even when properly designed and meta-tagged, will not realize full reusability without the full benefits of the semantic web. Aiming to close the gap between knowledge management and e-learning through the integration of different knowledge components, the knowledge map approach is adopted to enable the visualization of knowledge representation, and the personalization of learning experience.

\section{Knowledge Personalization}

Learning is often described as an ongoing cycle, occurring as a sequence of phases: first, concrete experiences generate an opportunity for observation and reflection; this, in turn, leads to the creation of new concepts and models that are then tested in novel situations; etc.

People need four different types of skills to make their learning cycle effective; more specifically, they have to:

- engage openly and in new experiences,

- reflect and observe their experiences from many perspectives,

- create concepts that integrate observations and,

- use these theories in decision making and problem solving.

In many important learning models, learning starts when the person experiences a practical or a cognitive dissonance. Then routine action breaks down, the learner realizes that active sense-making is needed, and the world needs to be reconstructed. This reconstruction may require reorganization of meaning and also reconfiguration of the material environment. In classroom settings, this process can be simulated by problembased learning situations, where the student is presented with a specific construction of the world, for example using a textual description, and the dynamics of the world is shown to lead to a contradiction or a problem that needs to be solved. Students may also collaborate in solving the problem, for example, by taking different roles and presenting different interpretations of the situation. Such problem-based learning settings can be enhanced by immersive information environments where the learner can effectively experience cognitive dissonance and where problem-solving resources are readily available. Our architecture specifically addresses the special requirements imposed in these information environments such as business environments, where tacit 
knowledge, is highly subjective in nature, as it is developed by an individual based on his cognitive and conceptual models of external processes. This organizational tacit knowledge should be defined and structured in a manner enabling further formulation and categorization providing reusability and communication.

However, apart from defining knowledge and its structure, it is important to address knowledge personalization and visualization in any e-learning context. To tackle the problems of knowledge personalization, we follow as a general blueprint the notion of knowledge concept maps in our context referred as knowledge maps. The potential of concept maps as instructional tools and learning personalization via use of concept maps has been proposed previously [3], [4], [5], [6]. Knowledge maps are used as the graphical representations of knowledge to depict both the learning concepts and the relationships between them in a human - oriented approach. Graphically, the knowledge maps consist of nodes and labelled lines that represent some important aspect of a learner's propositional knowledge in a subject domain. In our proposed architecture knowledge maps are technically represented by ontologies. The content is organized in a knowledge base that contains ontologies that define the learning objects semantics and workflows. These ontologies are defined by the tutors with the usage of the design toolkit. The content is stored into the content database, which is a semantic repository that enables semantic queries from the e-learning services.

An ontology is a semantically enriched data model that represents a set of concepts within a domain and the relationships between those concepts [7]. It provides a common vocabulary to refer to the concepts of a domain, specifies relationships using logical statements that describe how the concepts are related and provides also rules for combining concepts and their relations to define extensions to the vocabulary.

The semantic web is an extension of the current web, whereby information is given well-defined meaning, to enable computers and people to work cooperatively [8]. The Web Ontology Language OWL is a semantic markup language for publishing and sharing ontologies on the World Wide Web. It is the most recent development in standard ontology languages, certified by the World Wide Web Consortium (W3C) to promote the Semantic Web vision. OWL is used by applications that need to process the content of information instead of just presenting information to humans. OWL goes beyond the languages XML and RDF in their ability to represent machine interpretable content on the Web, because it has more facilities for expressing meaning and semantics than them [9].

\section{Proposed Solution}

The design of experiential learning systems requires insights, concepts, technologies, and methodologies from a host of disciplines that often have limited dialogue with one another [10]. This dialogue and collaboration takes time, commitment, and considerable effort, but can ultimately result in a "hybridized theory and practice", capable of addressing problems that no single discipline or cluster of related disciplines can attempt alone.

Prior research in communications theory and the phenomenology of lived and mediated experience provide us with frameworks for understanding how to structure data so as to affect the experiences that human minds create when they encounter these structured data. Assimilating and taking advantage of such data requires recognition 
of their multimedia nature, the development of semantic models across different media, the representation of complex relationships in the data (such as spatio-temporal, causal, or evolutionary), and finally, the development of paradigms to mediate usermedia interactions [11]. To help generate insights from multiple heterogeneous data sources, any environment should allow users apply their senses directly to observe data and manage the information related to a particular event [12].

Our architectural approach contributes towards the proliferation of high quality learning environments, through the establishment of a framework for inquiry-based eLearning, exploiting the emerging paradigm of service-orientated architecture in a semantically web-based environment. In this context, our conceptual platform aims at exploiting the content of personnel education in business environments. This platform facilitates the management of content and the creation of courses and supports a number of additional e-learning services, as shown in Figure 1, which depicts the architecture of the proposed solution.

The platform is operated by three different communities of users, namely:

- the educational content providers that design educational programmes in the form of maps,

- the personnel that participate in the learning environment, accessing maps for their education, and

- the content providers

For the system to support the functionality of these three communities, the platform is divided into three sub systems, which are described below:

- e-learning subsystem: a portal-based e-learning environment that enables the three communities to collaborate through a set of structured learning objects. The learning objects are accessed though a set of e-learning services that wrap semantics and workflows of the information.

- Content and learning objects subsystem: the content is organized in a knowledge base that contains ontologies that define the learning objects semantics and workflows. The ontologies are defined by the tutors with the usage of the design toolkit. The content is stored into the content database, which is a semantic repository that enables semantic queries from the e-learning services; and interoperability services: a set of web services that enable collaboration with external organizations and/or repositories.

The platform network shall function as an integrated, context-sensitive, adaptable and interoperable educational environment, based on the concept of technology-enhanced experiential learning for competency, skills and performance enhancement.

In the core of the proposed pedagogical approach, lies the inquiry-based learning implemented as case-based learning (CBL) methodology in ill-structured domains. The whole learning experience is situated within the context of an "extended" learning community [13], [14]. By "extended" we mean that the community becomes a bridge between learners and the field of practice, motivating field professionals to act as "contributors", that is, providers of "raw" learning material emerging from their experience. This material, after undergoing appropriate didactical transformation, can become learning material in the form of advice-cases and learning scenarios, enabling 


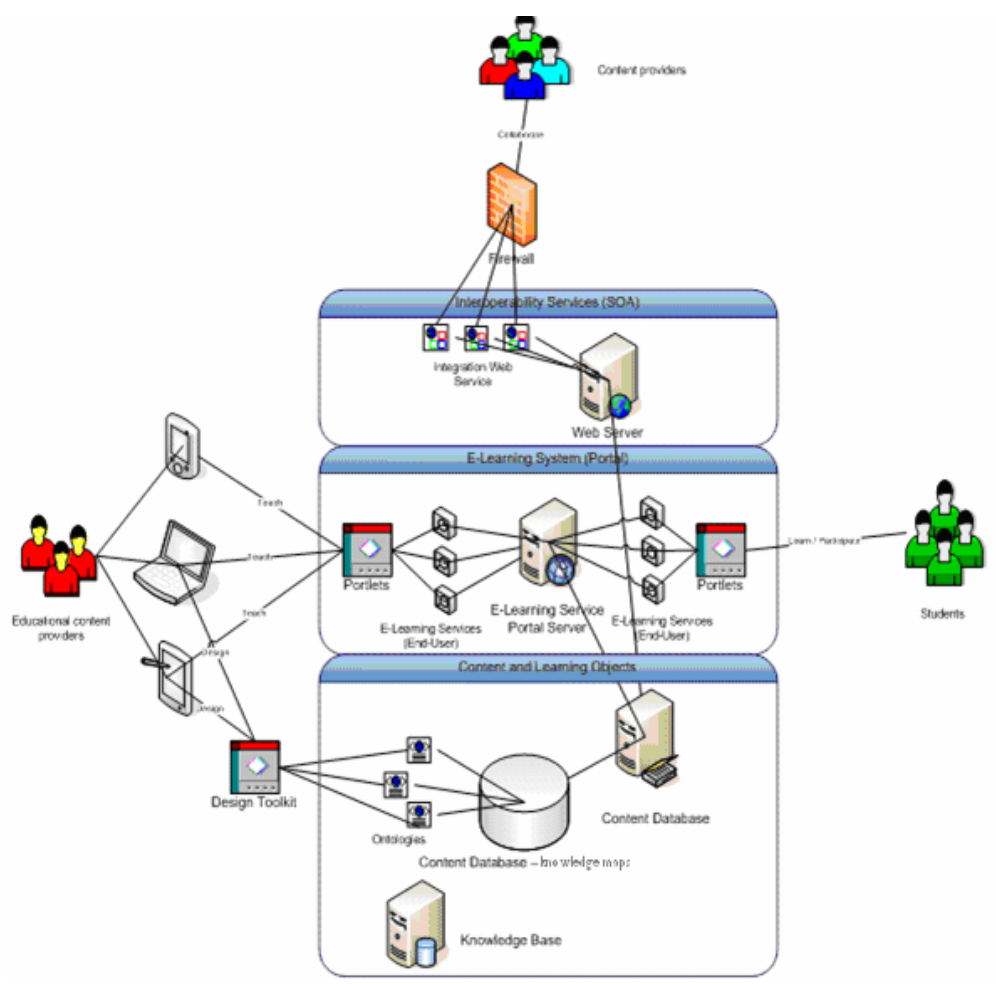

Fig. 1. Proposed architecture

learners to experience the complex and demanding situations encountered in the professional context. From a cognitive point of view our approach emphasizes two critical instructional interventions: "criss-crossing" of the learning material and learner scaffolding.

"Criss-crossing" (a basic tenet of cognitive flexibility theory) refers to guiding the learners through the informational landscape of case-based material, helping them to review relevant information from multiple perspectives. Case-based learning (CBL) is a widely acknowledged pedagogical approach for introducing personnel in the intricacies of ill-structured domains [15]. Cases are narrative structures, which immerse personnel in the context of real-world situations filled with "complexity, uncertainty, instability, uniqueness, and value conflict" [16]. By analyzing case-based material from various perspectives, personnel are expected to develop those flexible cognitive schemata that are necessary for ill-structured problem solving and knowledge transfer. To achieve this, cognitive flexibility theory prescribes that learners should thematically "criss-cross" the domain landscape studying the case material from different conceptual perspectives, in rearranged contexts, and for different purposes [17].

Scaffolds are instructional interventions that aim to help learners develop deeper understandings which might not be within their immediate grasp. Questioning strategies (including learners' self-generated questions) have been reported to significantly improve learning outcomes. In reviewing the literature [18], concludes that questioning 
strategies help personnel in important cognitive functions, such as focusing attention, stimulating prior knowledge, enhancing comprehension, and facilitating problemsolving processes.

Using the system the contributors may upload and index their material, using a domain specific indexing scheme. Pedagogical content providers ("instructors") can prepare scenarios for learners to work with, construct "paths" to help them criss-cross relevant cases and provide useful cognitive scaffolds (for example, in the form of question prompts) to help them reflect on the material. Learners, finally, can work on assigned scenarios at two levels of expertise (novice or advanced learner), which implies different access privileges and level of difficulty of assigned tasks. Drawing on the above theoretical background, we suggest that four fundamental content organization features in the environment are: the scenario, the learning case, the path and the scripts.

Scenario: In Architecture the learners study by exploring various scenarios. A scenario is the main study unit, presenting to learners a plausible problem-case. Learners are engaged in decision-making process, playing the role of practitioners, who face some open-ended questions (scenario questions) regarding critical decisions of the field experience presented in the scenario.

Advice-cases: In order to successfully deal with the scenario questions, learners need to reflect on decisions taken and practices implemented in other similar situations. Such information is offered to them through criss-crossing of learning advicecases. These cases present field experiences with some specific outcome (failure or success). Usually it is illustrated how effective or inefficient decisions or actions has resulted to respectively successful or unsuccessful management of the problematic situation. Each advice-case is divided into "case-frames", which are smaller parts of the case presenting some meaningful and self-contained aspect of it. The content of a case-frame refers to the impact of one domain factor (theme) on the specific case, thus enabling the conceptual indexing of the case material. Contributors of the learning material will be able to add conceptual indexing factors depending on their common understanding of the domain, thus collaboratively developing a domain-specific metadata scheme to index the learning material.

Path: Paths are sequences of case-frames from various cases, guiding personnel through past field experiences (advice-cases) and focusing on specific issues. A path, for example, may illustrate how a specific emergency situation was handled (with or without success) in a number of cases. The instructor can flexibly construct a path by assembling any case-frames she likes, depending on the learning objective of the scenario. Each scenario is accompanied by a number of paths guiding learners to crisscross the material and reviews it from various perspectives (domain factors).

Scaffolding and Scripting: To engage learners in active processing of the material, learner scaffolds (in the form of question prompts) and collaboration scripts will be employed to guide both the learner-content and learner-learner interactions. Regarding the former, a specific questioning scheme will appear each time a case-frame is selected for study. These questions ask learners to: (a) identify concrete events/issues that play important role in the situation, (b) recall other instances, where similar evens/issues were encountered, and (c) state some useful conclusions regarding their expected successful performance (decision taking). 
After submitting their answers learners get system feedback in the form of hints that help them self-assess their answers and practices. Regarding the latter (learnerlearner interaction), a collaboration script will be used to guide learners on how to collaborate productively. Collaboration scripts [19], are didactic scenarios providing specific instructions for small groups of learners on what activities need to be executed, when they need to be executed, and by whom they need to be executed in order to foster individual knowledge acquisition.

\section{Conclusions}

In this paper we shed light on these issues of knowledge personalization and visualization in e-learning systems focusing in education in business environments. We argued that the focus should be on transforming the experience held as tacit knowledge into structured formulated and reusable piece of explicit knowledge. For this we propose an architecture presenting the proposed e-learning system. Our system architecture makes use of a knowledge model to facilitate continuous dialogue and inquiry-based knowledge learning in business environments. This architecture offers a knowledge environment that represents the semantic web version of e-learning, aiming to close the gap between knowledge management and e-learning through the employment of graphical representations of knowledge, depicting both the learning concepts and the relationships between them in a human - oriented approach.

\section{References}

1. Miltiadis, D.L., Pouloudi, N.: E-learning: Just a waste of time. In: Strong, D., et al. (eds.) Proceedings of the Seventh Americas Conference on Information Systems (AMCIS 2001), Boston, MA, August 3-5, pp. 216-222 (2001)

2. McCalla, G.: The Ecological Approach to the Design of E-Learning Environments: Purpose-based Ca p t u re and Use of Information About Learners. Journal of Interactive Media in Education (2004)

3. Teo, C.B., Gay, K.L.: Concept-based system design to personalize E-learning. WSEAS Trans. Inf. Science Applications 1(5), 1248-1255 (2004b)

4. Teo, C.B., Gay, K.L.: Concept map provision for E-learning. Int. J. Instructional Technology Distance Learning 3(7), 17-32 (2006a)

5. Teo, C.B., Gay, K.L.: Provision of self-directed learning using concept mapping. WSEAS Trans. Advances Eng. Education 3(6), 491-498 (2006b)

6. Teo, C.B., Gay, K.L., Chang, S.C.: Pedagogy considerations for E-learning. Int. J. Instructional Technology Distance Learning 3(5), 3-26 (2006b)

7. Antoniou, G., Van Harmelen, F.: A Semantic Web Primer. MIT Press, Cambridge (2004)

8. Berners-Lee, T., Hendler, J., Lassila, O.: The semantic web. Scientific American (2001)

9. McGuinness, D.L., Van Harmelen, F.: OWL Web Ontology Language Overview. W3C Recommendation. MIT Press, Cambridge (2004)

10. Davis, M.: Theoretical Foundations for Experiential Systems Design. In: Proceedings of ETP 2003, Berkeley, California, USA, pp. 45-52 (2003) 
11. Singh, R., Knickmeyer, R., Gupta, P., Jain, R.: Designing Experiential Environments for Management of Personal Multimedia. In: Proceedings of MM 2004, New York, New York, USA, October 10-16, pp. 496-499 (2004)

12. Jain, R.: Experiential Computing. Communications of the ACM 46(7), 48-54 (2003)

13. Demetriadis, S., Barbas, A., Molohides, A., Palaigeorgiou, G., Psillos, D., Vlahavas, I., Tsoukalas, I., Pombortsis, A.: Cultures in Negotiation: Teachers' Acceptance/Resistance Attitudes Considering the Infusion of Technology into Schools. Computers \& Education 41(1), 19-37 (2003)

14. Demetriadis, S., Barbas, A., Psillos, D., Pombortsis, A.: Introducing ICT in the Learning Context of Traditional School: what is transformed and why. In: Vrasidas, C., Glass, V.G. (eds.) Current Perspectives on Applied Information Technologies II, pp. 99-116. Information Age Publishing, Charlotte (2004)

15. Jonassen, D.H., Hernandez-Serrano, J.: Case-Based Reasoning and Instructional Design: Using Stories to Support Problem Solving. ETR\&D 50(2), 65-77 (2002)

16. Schön, D.A.: The Reflective Practitioner - How Professionals Think in Action. Basic Books, New York (1993)

17. Spiro, R.J., Jehng, J.: Cognitive flexibility and hypertext: Theory and technology for the non-linear and multidimensional traversal of complex subject matter. In: Nix, D., Spiro, R. (eds.) Cognition, Education, and Multimedia. Ehrlbaum, Hillsdale (1990)

18. Ge, X.: Scaffolding personnel' problem-solving processes on an ill-structured task using question prompts and peer interactions. Ph.D. Thesis. Pennsylvania State University, Philadelphia (2001)

19. Weinberger, A., Stegmann, K., Fischer, F., Mandl, H.: Scripting argumentative knowledge construction in computer-supported learning environments. In: Fischer, F., Kollar, I., Mandl, H., Haake, J. (eds.) Scripting computer-supported collaborative learning: Cognitive, computational and educational perspectives, pp. 191-211. Springer, New York (2007) 